\title{
Effects of 5-fluorouracil and class III phosphoinositide 3-kinase small interfering RNA combination therapy on SGC7901 human gastric cancer cells
}

\author{
BAO-SONG ZHU ${ }^{1 *}$, JIA-LEI SUN ${ }^{2 *}$, WEI GONG ${ }^{1 *}$, XING-DING ZHANG ${ }^{3}$, \\ YONG-YOU WU ${ }^{1}$ and CHUN-GEN XING ${ }^{1}$
}

\author{
${ }^{1}$ Department of General Surgery, The Second Affiliated Hospital, Soochow University, Suzhou, Jiangsu 215004; \\ ${ }^{2}$ Department of General Surgery, The First Changshu Affiliated Hospital, Soochow University, Changshu, Jiangsu 215500; \\ ${ }^{3}$ Cyrus Tang Hematology Center, Soochow University, Suzhou, Jiangsu 215123, P.R. China
}

Received November 18, 2013; Accepted October 1, 2014

DOI: $10.3892 / \mathrm{mmr} .2014 .2926$

\begin{abstract}
The aim of the present study was to investigate the effects of small interfering RNA-mediated inhibition of Class III phosphoinositide 3-kinase (PI3K) signal transduction on the proliferation, apoptosis and autophagy of SGC7901 gastric cancer cells. The present study also aimed to examine the contribution of autophagic inhibition to the antitumor effects of 5-fluorouracil (5-FU). A PI3K(III)-RNA interference (i)-green fluorescent protein (GFP) recombinant replication adenovirus (AD) and the negative control (NC)-RNAi-GFP control AD were constructed and infected into SGC7901 cells. A methyl thiazolyl tetrazolium assay was used to determine the growth rate of the SGC7901 cells. Immunofluorescent staining was used to detect microtubule-associated protein 1 light chain 3 expression. The mitochondrial membrane potential was measured using the JC-1 fluorescent probe. Autophagic expression was monitored with MDC staining and transmission electron microscopy. The results revealed that following combination treatment of the SGC7901 gastric cancer cells with 5-FU + PI3K(III)-RNAi-AD, the optical density absorbance values at 24,48 and $72 \mathrm{~h}$ were $0.17 \pm 1.64,0.13 \pm 4.64$ and $0.11 \pm 3.56 \%$, respectively, with cell viability inhibition ratios of $45.89 \pm 6.67,72.57 \pm 9.48$ and $87.51 \pm 4.65 \%$, respectively. As compared with the other treatment groups, the inhibition rate in the combined treatment group was significantly
\end{abstract}

Correspondence to: Professor Chun-gen Xing, Department of General Surgery, The Second Affiliated Hospital of Soochow University, 1055 San Xiang Road, Suzhou, Jiangsu 215004, P.R. China

E-mail: xingcg@126.com

${ }^{*}$ Contributed equally

Key words: 5-fluorouracil, class III phosphoinositide 3-kinase, small interfering RNA, apoptosis, autophagy higher $(\mathrm{P}<0.05)$. The percentages of the cells with green fluorescence in the combined treatment group were $74.4 \pm 3.86$ (24 h), 82.3 $\pm 1.84(48 \mathrm{~h})$ and $92.5 \pm 1.1 \%$ (72 h), which were larger than those of the other groups. The percentage of cells with green fluorescence became larger, which indicated that the mitochondrion membrane potential had been reduced to a greater extent. MDC staining revealed that the number of autophagic vacuoles in the cells (measured at 24, 48 and $72 \mathrm{~h}$ ) decreased gradually with time, with more autophagic vacuoles observed in the cells in the control group at $24 \mathrm{~h}$ than those in the other treatment groups. Fewest autophagic vacuoles were identified in the combined treatment group. Using a fluorescence microscope, the immune fluorescence expression of microtubule-associated proteins 1A/1B light chain $3 \mathrm{~A}$, which is the specific protein of autophagy, in the combined treatment group was observed to be significantly downregulated, as compared with the other groups. As determined by transmission electron microscopic observation of the SGC7901 gastric cancer cells, the degree of autophagy in the combined treatment group was significantly reduced, as compared with that of the other treatment groups. In conclusion, following combined treatment with 5-FU and an inhibitor of class III PI3K signal transduction, the proliferation of SGC7901 cells was significantly suppressed, the mitochondrion membrane potentials were significantly reduced and the expression levels of autophagic markers were significantly downregulated.

\section{Introduction}

Gastric cancer is the fourth most common type of cancer and the second leading cause of cancer-associated mortality worldwide (1), with approximately one million new cases diagnosed each year. The phosphoinositide-3 kinases (PI3Ks) are a family of enzymes that have been categorized into three classes, class I, II and III. Class III PI3K is constitutively active and is able to generate phosphatidylinositol 3-phosphate from phosphatidylinositol (2-4). Various studies have shown that the class III phosphatidylinositol 3-kinase signaling pathways are the central conduit in the regulation of autophagy (5). 
Previous studies have indicated that autophagy is involved in tumor cell resistance to chemotherapy and that inhibition of autophagy enhances the cytotoxicity of certain chemotherapeutic agents (6,7). Autophagy is an important mechanism in various physiopathological processes, including tumorigenesis, development, cell death and survival $(8,9)$. Autophagy has also been demonstrated to have a complex association with apoptosis, with an important role in promoting cell survival against apoptosis (10). The induction of cell death and the inhibition of cell survival are the main principles of cancer therapy. Resistance to chemotherapeutic agents is a major problem in oncology and limits the efficacy of anticancer drugs.

The identification of Class III PI3K revealed a novel role for autophagy in inducing cell death, and Class III PI3K is considered to be a crucial modulator of apoptosis and autophagy. In the present study, the effects of PI3K(III)-RNA interference (i)-green fluorescent protein (GFP) adenovirus (AD) on the growth and apoptosis of gastric cancer cells in vitro were analyzed. The effects of the PI3K(III)-RNAi-GFP AD on the activation of apoptosis and autophagy, and the contribution of autophagy to chemosensitivity effects of 5-fluorouracil (5-FU) in the SGC7901 gastric cancer cell line were investigated.

\section{Materials and methods}

Reagents. SGC7901 gastric cancer cells were obtained from the Shanghai Institute of Cell Biology, Chinese Academy of Sciences (Shanghai, China). RPMI-1640 medium was provided by Gibco-BRL (Rockville, MD, USA). Fetal calf serum (FCS) was purchased from Hangzhou Sijiqing Biological Engineering Material Co., Ltd. (Hangzhou, China). L-glutamine and methyl thiazolyl tetrazolium (MTT) were provided by Sigma-Aldrich (St. Louis, MO, USA).

Adenoviral vectors and infections. An RNAi sequence against Class III PI3K was designed and vectors that expressed Class III PI3K small interfering (si)RNA were constructed. A Class III PI3K-specific target sequence was selected with the Invitrogen online siRNA tool (http://www.invitrogen. com/rnai) using the Class III PI3K reference sequence (GenBank accession no. NM_002647). The target sequence was as follows: Class III PI3K (base 2521-2641), 5'-TCCGCT TAGACCTGTCGGATGAAGAGGCT-3'. Subsequently, the siRNA was chemically synthesized and a lentiviral vector was constructed. The insertion of the specific siRNA was further verified by sequencing. The siRNA were sequenced on the SOLiD 5500xl system (35 bp read length; Shanghai Genesil Co., Ltd., Shanghai, China). The recombinant AD vector that expresses siRNA against Class III PI3K was synthesized by Shanghai Genesil Co., Ltd. The replication-defective adenoviral vectors expressing GFP (PI3K(III)-RNAi-GFP-AD and control negative control (NC)-RNAi-GFP-AD) were stored at $-80^{\circ} \mathrm{C}$. Infections were performed at $70-75 \%$ confluence in Dulbecco's modified Eagle's medium supplemented with $2 \%$ FCS. The cells were subsequently incubated at $37^{\circ} \mathrm{C}$ for a minimum of $4 \mathrm{~h}$, followed by the addition of fresh medium. The cells were subjected to functional analysis at fixed time points following infection as described for the individual experimental conditions.
Drug preparation. 5-FU (Sigma-Aldrich) was diluted in saline to produce a stock solution that was stored according to the manufacturers' instructions. The 5-FU concentration selected for use in the experiments was $2 \mathrm{mg} / \mathrm{l}$, in accordance with the manufacturer's instructions.

Determination of optimal multiplicity of infection (MOI). A total of $1 \times 10^{4}$ SGC7901 cells/well were seeded in 96-well plates to produce $60-70 \%$ cultured adherent cells. Subsequently, 10, 20, 30, 50 and $100 \mathrm{MOI}$ of the NC-RNAi-GFP-AD was added to $100 \mu \mathrm{l}$ diluted infected cells, $8 \mathrm{~h}$ after the addition of RPMI 1640 culture medium with $10 \%$ fetal bovine serum. The cells were cultured for $48 \mathrm{~h}$ then counted using a fluorescence microscope (Leica DMI4000B; Leica Microsystems Wetzlar GmbH, Wetzlar, Germany) to calculate the number of cells that expressed GFP.

Cell culture and MTT assay. The SGC7901 cells were maintained in RPMI 1640 medium containing 10\% heat-inactivated fetal bovine serum and $0.03 \%$ L-glutamine, and were incubated in a $5 \% \mathrm{CO}_{2}$ atmosphere at $37^{\circ} \mathrm{C}$. Cell viability was evaluated using an MTT assay. To analyze the response to PI3K(III)-RNAi-GFP-AD, the cells were plated into 96-well microplates $\left(7 \times 10^{4}\right.$ cells/well) and the PI3K(III)-RNAi-GFP-AD was added to the culture medium. MTT solution was then added to the culture medium (500 $\mu \mathrm{g} / \mathrm{ml}$ final concentration) for $4 \mathrm{~h}$ prior to the end of treatment and the reaction was terminated by the addition of $10 \%$ acidic sodium dodecyl sulfate $(100 \mu \mathrm{l})$. The optical density of each well was determined using a microculture plate reader (Bio-Rad Laboratories, Richmond, CA, USA) at $570 \mathrm{~nm}$. Each absorbance value was normalized to the survival percentage value. Each assay was performed in triplicate.

Visualization of monodansylcadaverin (MDC)-labeled autophagic vacuoles. The SGC7901 cells were plated on 24-chamber culture slides, cultured for $24 \mathrm{~h}$ and then incubated with 5-FU, adenovirus PI3K(III)-RNAi-GFP and control adenovirus NC-RNAi-GFP treatment in RPMI 1640 with $10 \%$ FCS for 12 or $24 \mathrm{~h}$. The autophagic vacuoles were labeled with MDC (Sigma-Aldrich) as described previously (11), by incubating the cells with $0.001 \mathrm{mmol} / 1 \mathrm{MDC}$ in RPMI 1640 at $37^{\circ} \mathrm{C}$ for $10 \mathrm{~min}$. Following incubation, the cells were washed twice with phosphate-buffered saline (PBS) and immediately analyzed under a fluorescence microscope (Nikon Eclipse TE 300; Nikon Corporation; Tokyo, Japan). All experiments were repeated in triplicate.

Detection of the microtubule-associated proteins $1 A / 1 B$ light chain 3A (MAP1LC3) protein by immunofluorescence. MAP1LC3 immunodetection was performed on SGC7901 cells treated with PI3K(III)-RNAi-GFP-AD or 5-FU, and mounted onto silanized slides. The cells were cultured on sterilized glass coverslips, fixed with $4 \%$ paraformaldehyde and blocked with $0.1 \%$ bovine serum albumin in PBS. The slides were then incubated with a rabbit monoclonal anti-human antibody against LC3 (dilution, 1:150; cat. no. 12741; Cell Signaling Technology, Inc., Beverly, MA, USA). Following washing, the slides were incubated with a secondary ghost monoclonal anti-rabbit cy3 immunoglobulin G (IgG)-conjugated antibody 
A

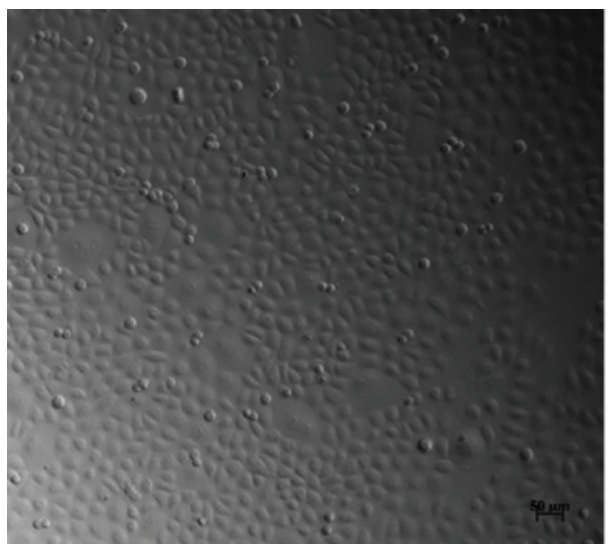

C

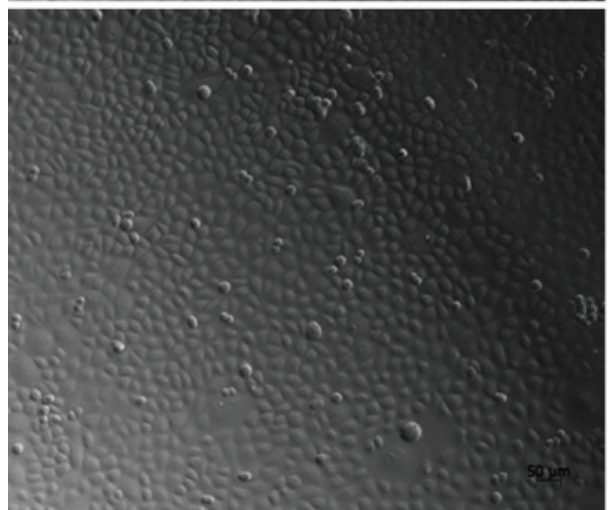

B

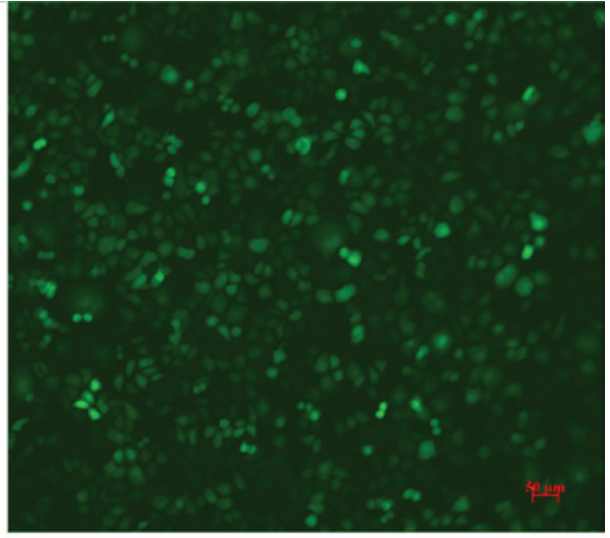

D

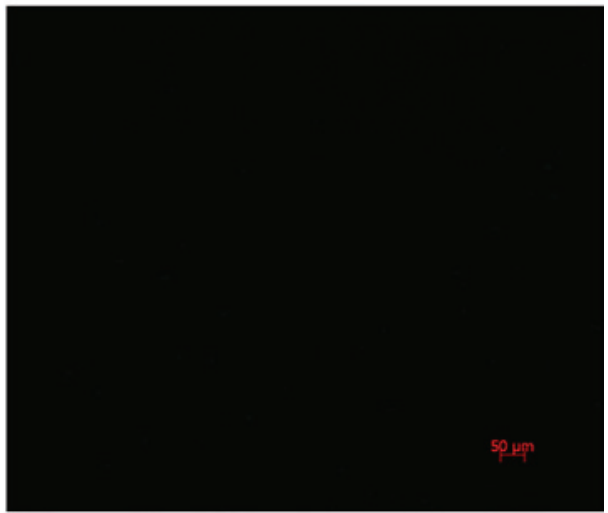

Figure 1. Transfection efficiency and cell morphology following PI3K(III)-RNAi-GFP(30 MOI) and NC-RNAi-GFP(30 MOI) adenoviral treatment, as detected using fluorescence microscopy. SGC7901 gastric cancer cells were incubated with PI3K(III)-RNAi-GFP adenovirus (30 MOI) for 24, 48 and 72 h. (A) Control cells infected with NC-RNAi-GFP adenovirus. (B) $24 \mathrm{~h}$ after PI3K(III)-RNAi-GFP adenovirus (30 MOI) treatment. (C) 48 h after PI3K(III)-RNAi-GFP adenovirus (30 MOI) treatment. (D) $72 \mathrm{~h}$ after PI3K(III)-RNAi-GFP adenovirus (30 MOI) treatment. Magnification, x200; n=3. PI3K, phosphoinositide 3-kinase; RNAi, RNA interference; GFP, green fluorescent protein; MOI, multiplicity of infection; NC, negative control.

(1:500; cat. no. SAB3700843; Sigma-Aldrich). Cell nuclei were counter-stained with $1 \mu \mathrm{g} / \mathrm{ml}$ Hoechst 33258 (Keygen Biotechnology Co., Ltd., Nanjing, China). and the slides were treated with fluorescent mounting medium (Dako, Carpinteria, CA, USA). Images were captured using a laser confocal microscope (Leica, Mannheim, Germany).

Mitochondrial membrane potential $(\Delta \Psi)$. Alterations in the mitochondrial membrane potential were the earliest indication of cell death but not the distinction between apoptosis and oncosis. The mitochondrial membrane potential of the SC7901 cells was measured using a KeyGEN Mitochondrial Membrane Sensor kit (Nanjing KeyGen Biotech. Co. Ltd., Nanjing, China). The cells were stained and fluorescence intensity was detected with flow cytometry (FCM; Partec, Münster, Germany).. The mitochondrial $\Delta \Psi$ was calculated from the fluorescence intensities using CellQuest ${ }^{\mathrm{TM}}$ software (Becton Dickinson, Bedford, MA, USA) (12).

Transmission electron microscopy (TEM). All reagents for these experiments were obtained from Keygen Biotechnology Co., Ltd. For TEM, the SGC7901 cells were fixed in 2\% glutaraldehyde in $0.1 \mathrm{M}$ sodium cacodylate buffer $(\mathrm{pH} 7.0)$ for $2 \mathrm{~h}$, post-fixed in $2 \%$ osmium tetroxide for $2 \mathrm{~h}$ and then washed with $0.1 \mathrm{M}$ cacodylate buffer. The cells were coated in 5\% Noble Agar and washed with distilled water five times, with further fixing in $2 \%$ uranyl acetate for $2 \mathrm{~h}$, followed by dehydration in $50 \%$ (15 $\mathrm{min}), 70 \%$ (16 h), 85\% (15 min),
95\% (15 min) and two incubations in $100 \%$ ethanol, $15 \mathrm{~min}$ each. The cells were subsequently cleared by two incubations in propylene oxide, each $15 \mathrm{~min}$, and infiltrated with epon resin:propylene oxide $(1: 1)$ for $3 \mathrm{~h}$, epon resin:propylene oxide (3:1) for $16 \mathrm{~h}$ and two incubations with pure epon resin for a total of $6 \mathrm{~h}$. Thin sections were mounted onto grids and examined under an electron microscope (Philips CM120; Koninklijke Philips N.V., Amsterdam, The Netherlands) (13).

Statistical analysis. The results are presented as the mean \pm standard deviation. Statistical analysis was performed using the Dennett's test and analysis of variance. $\mathrm{P}<0.05$ was considered to indicate a statistically significant difference.

\section{Results}

Transfection efficiency and cell morphology. Following treatment of the SGC7901 cells with PI3K(III)-RNAi-GFP-AD (30 MOI) for $24 \mathrm{~h}$, the cell body was observed to be swollen and rounded. The cells showed further deformation after $48 \mathrm{~h}$. Fragments by recombinant AD containing GFP, after $72 \mathrm{~h}$ transfection, the SGC7901 cells were counted using a fluorescence microscope hair green fluorescence of tumor cells (Fig. 1). At 30 MOI, the transfection efficiency was revealed to be $95 \pm 2.4 \%$.

PI3K(III)-RNAi-GFP-AD inhibits cell viability and enhances 5-FU-mediated tumor cell growth inhibition. The 


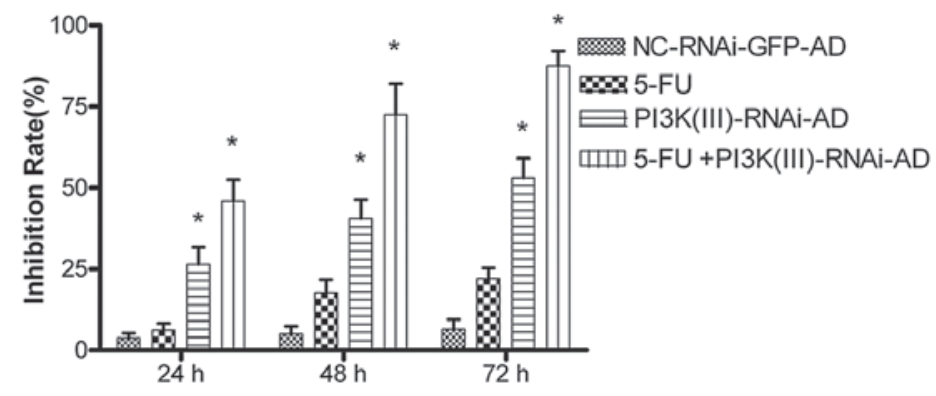

Figure 2. Reduced viability of SGC7901 gastric cancer cells is observed following PI3K(III)-RNAi-EGFP-AD or 5-FU treatment. SGC7901 cells $\left(7 \times 10^{4}\right.$ cells $\left./ \mathrm{ml}\right)$ were cultured with PI3K(III)-RNAi-EGFP-AD or 5-FU for the indicated time periods and cell viability was analyzed using a MTT assay. PI3K(III)-RNAi-EGFP-AD exerted a greater effect when used in combination with 5-FU than when used alone. PI3K(III)-RNAi-EGFP-AD inhibited the proliferation of SGC7901 gastric cancer cells and enhanced the chemosensitivity of the cells to 5-FU. Values are presented as the mean \pm standard deviation of three independent experiments. "P<0.05 compared with the NC-RNAi-GFP-AD alone treatment group. PI3K, phosphoinositide 3-kinase; RNAi, RNA interference; EGFP, enhanced green fluorescence protein; 5-FU, 5-fluorouracil; MOI, multiplicity of infection; NC, negative control; AD, adenovirus.

PI3K(III)-RNAi-GFP-AD reduced SGC7901 cell viability in a time-dependent manner. The MTT assays revealed that after $24 \mathrm{~h}$ of PI3K(III)-RNAi-GFP treatment, the rate of inhibition reached $26.45 \pm 11.23 \%$ at $30 \mathrm{MOI}$. The rate of inhibition rose when the incubation time was prolonged, reaching $40.58 \pm 6.54 \%$ at $48 \mathrm{~h}$ and $53.12 \% \pm 2.0 \%$ at $72 \mathrm{~h}$ after treatment (Fig. 2). In order to assess the clinical value of the PI3K(III)-RNAi-GFP-AD in tumor treatment and to analyze the synergistic inhibitory effect of the PI3K(III)-RNAi-GFP-AD on growth in combination with a chemotherapy drug, $2 \mathrm{mg} / \mathrm{l}$ of the 5 -FU chemotherapy drug was administered to the cells along with the PI3K(III)-RNAi-GFP. The PI3K(III)-RNAi-GFP-AD was demonstrated to exert a greater effect when used in combination with 5-FU than when used alone (Fig. 2). Following the treatment of SGC7901 gastric cancer cells with 5-FU + PI3K(III)-RNAi-AD, the absorbance values at 24,48 and $72 \mathrm{~h}$ were $0.17 \pm 1.64$, $0.13 \pm 4.64$ and $0.11 \pm 3.56 \%$, respectively, with inhibition ratios at $45.89 \pm 6.67,72.57 \pm 9.48$ and $87.51 \pm 4.65 \%$, respectively. In the combined treatment group, as compared with other groups, the inhibition rate was significantly higher $(\mathrm{P}<0.05)$. Thus, PI3K(III)-RNAi-GFP-AD treatment inhibited the proliferation of SGC7901 gastric cancer cells and enhanced the chemosensitivity of the cells to 5-FU.

PI3K(III)-RNAi-GFP-AD transfection and 5-FU treatment reduce the number of autophagic vacuoles. The autofluorescent substance MDC has been recently shown to be a marker for late autophagic vacuoles (11). To investigate the autophagy-inhibiting effect of PI3K(III)-RNAi-GFP-AD transfection and 5-FU treatment of SGC7901 cells, the cells were stained with MDC. When the cells were viewed under a fluorescence microscope, MDC-labeled autophagic vacuoles appeared as distinct dot-like structures distributed in the cytoplasm or in the perinuclear area. The number of MDC-labeled vesicles in the SGC7901 cells was observed to be significantly fewer following treatment with PI3K(III)-RNAi-GFP-AD (30 MOI) or 5-FU for between 24 and $72 \mathrm{~h}$, as compared with the number of vesicles in the control group cells (Fig. 3). A reduction in the number of MDC-labeled vesicles following treatment with PI3K(III)-RNAi-GFP-AD and 5-FU was revealed, as compared with $\mathrm{X}$ used alone (Fig. 3).

PI3K(III)-RNAi-GFP-AD transfection and 5-FU treatment reduce punctate $L C 3$. To analyze whether autophagy was inhibited following exposure to PI3K(III)-RNAi-GFP-AD transfection or 5-FU in SGC7901 cells, evaluation of cyanine 3 (CY3)-LC3 modulation using fluorescent microscopic methods was employed. LC3 is a mammalian homologue of autophagy-related protein 8 (Atg8), a protein required for autophagy that is recruited to the autophagosomal membrane, which is routinely examined to monitor autophagy. CY3-LC3, LC3 fused to red fluorescent protein, measured as an elevation in punctate CY3-LC3, was detected with fluorescence microscopy. The results revealed that the PI3K(III)-RNAi-GFP-AD and 5-FU treatments reduced the punctate distribution of LC3 immunoreactivity, which indicated downregulation of autophagosome formation by PI3K(III)-RNAi-GFP-AD between 24 and $72 \mathrm{~h}$ (Fig. 4). The immune fluorescence expression levels of LC3, which is a specific autophagy protein, were significantly downregulated, as compared with other groups, in the combined treatment group, as determined by fluorescence microscopy. A greater inhibition of LC3 punctate distribution was observed following treatment with PI3K(III)-RNAi-GFP-AD and 5-FU than when $\mathrm{X}$ was used alone (Fig. 4).

PI3K(III)-RNAi-GFP-AD transfection and 5-FU treatment induce mitochondrial dysfunction. The mitochondrial membrane potential of the SGC7901 cells was detected using JC-1. Mitochondrial membrane potential $(\Delta \Psi)$ collapse was observed as early as $24 \mathrm{~h}$ after PI3K(III)-RNAi-GFP-AD (30 MOI) treatment reaching a maximum at $72 \mathrm{~h}$ (Fig. 5). The percentages of cells with green fluorescence in the combined treatment group were $74.4 \pm 3.86(24 \mathrm{~h}), 82.3 \pm 1.84(48 \mathrm{~h})$ and $92.5 \pm 1.1 \%(72 \mathrm{~h})$, which were larger than those in the respective other groups. The increase in the percentage of cells with green fluorescence indicated that the mitochondrial membrane potential was reduced to a greater extent. Mitochondrial membrane potential collapse indicates cell apoptosis or necrosis. PI3K(III)-RNAi-GFP-AD (30 MOI) induced mitochondrial dysfunction, activated cell apoptosis and enhanced the effects of 5-FU-mediated increased green fluorescence emission in the SGC7901 cells.

PI3K(III)-RNAi-GFP-AD and 5-FU treatment inhibits autophagy and impairs mitochondria. TEM was used to identify any ultrastructural changes in the SGC7901 cells following PI3K(III)-RNAi-GFP-AD (30 MOI) treatment. Control 


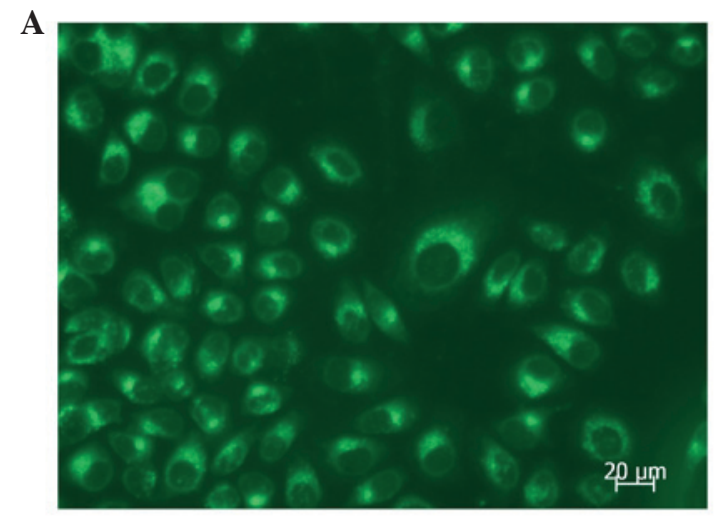

B

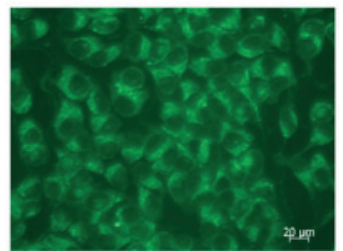

$\mathbf{F}$

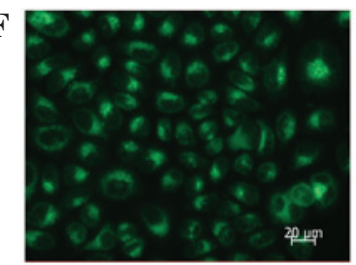

$\mathbf{J}$

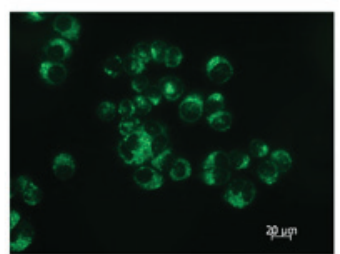

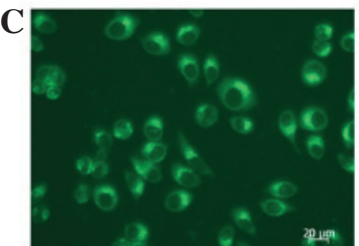

D

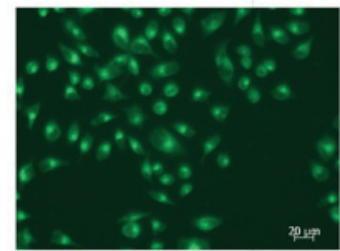

$\mathbf{E}$
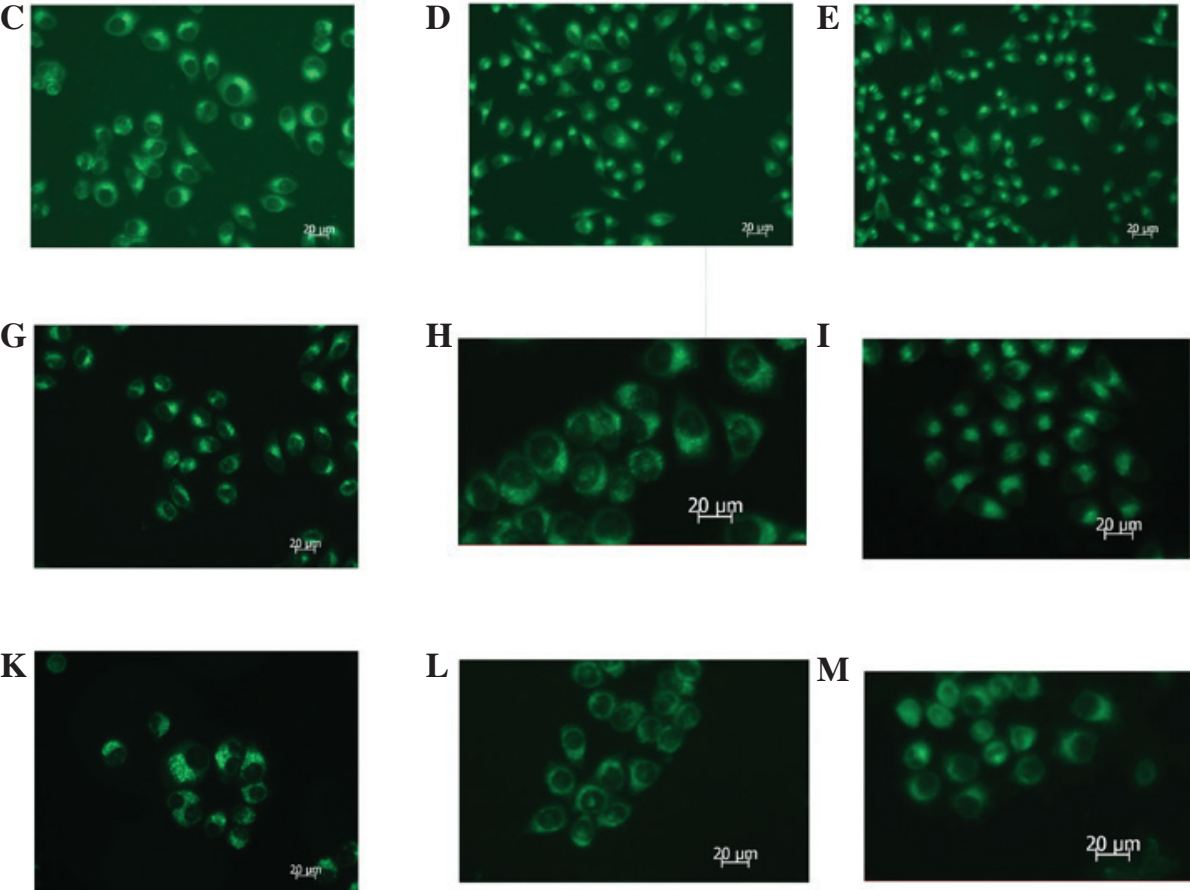

$\mathbf{L}$

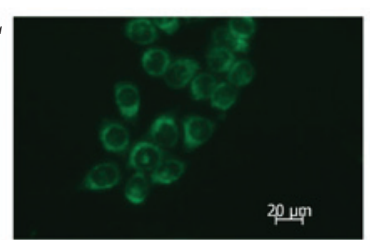

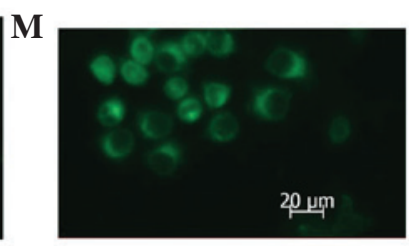

Figure 3. MDC staining reveals that autophagy is inhibited following PI3K(III)-RNAi-GFP adenovirus (30 MOI) or 5-FU treatment. SGC7901 gastric cancer cells were incubated with PI3K(III)-RNAi-GFP adenovirus (30 MOI), NC-RNAi-GFP adenovirus or 5-FU for the indicated time periods, and were then stained with MDC (100 $\mu \mathrm{mol} / 1)$. Fluorescent particles revealed late autophagic vacuoles. (A) $24 \mathrm{~h}$ after control treatment. (B) $24 \mathrm{~h}$ after NC-RNAi-GFP adenovirus treatment. (C) $24 \mathrm{~h}$ after 5-FU treatment. (D) $24 \mathrm{~h}$ after PI3K(III)-RNAi adenovirus treatment. (E) $24 \mathrm{~h}$ after 5-FU and PI3K(III)-RNAi adenovirus treatment. (F) $48 \mathrm{~h}$ after NC-RNAi-GFP adenovirus treatment. (G) $48 \mathrm{~h}$ after 5-FU treatment. (H) $48 \mathrm{~h}$ after PI3K(III)-RNAi adenovirus treatment. (I) $48 \mathrm{~h}$ after 5 -FU and PI3K(III)-RNAi adenovirus treatment. (J) $72 \mathrm{~h}$ after NC-RNAi-GFP adenovirus treatment. (K) $72 \mathrm{~h}$ after 5-FU treatment. (L) $72 \mathrm{~h}$ after PI3K(III)-RNAi adenovirus treatment. (M) $72 \mathrm{~h}$ after 5-FU and PI3K(III)-RNAi adenovirus treatment. Magnification, $\mathrm{x} 400$; n=3. MDC, monodansylcadaverin; PI3K, phosphoinositide 3-kinase; RNAi, RNA interference; GFP, green fluorescence protein; 5-FU, 5-fluorouracil; MOI, multiplicity of infection; NC, negative control.

cells exhibited a round shape and contained normal-looking organelles, nucleus and chromatin (Fig. 6A). However, subsequent to 5-FU treatment, inhibition of autophagy and apoptotic body induction were observed (Fig. 6C,G and K). PI3K(III)-RNAi-GFP-AD (30 MOI)-treated cells also exhibited inhibited autophagy and induced cell apoptosis, as shown in Fig. 6D,H and J. Following 5-FU and PI3K(III)-RNAi-GFP-AD (30 MOI) combined treatment, the effects of 5-FU-induced cell death were elevated (Fig. 6E, I and M). The loss of organelles and cytoplasm vacuolization was also observed when the incubation time was prolonged. The degree of autophagy in the combined treatment group was significantly reduced, as compared with the other treatment groups.

\section{Discussion}

In the present study, the small interfering RNA (siRNA) of class III PI3K was revealed to reduce viability, inhibit autophagy and induce apoptosis in SGC7901 gastric cancer cells, thus demonstrating the cytotoxic effects of the PI3K(III)-RNAi-GFP-AD. These findings indicate that using siRNA targeting molecules in the class III PI3K signaling pathway is a potential strategy for treating gastric cancer. In addition, PI3K(III)-RNAi-GFP-AD was also observed to enhance the effects of 5-FU in inducing cell death.

Resistance to anticancer drug-induced cell death is a predominant cause of cancer treatment failure. The majority 
A

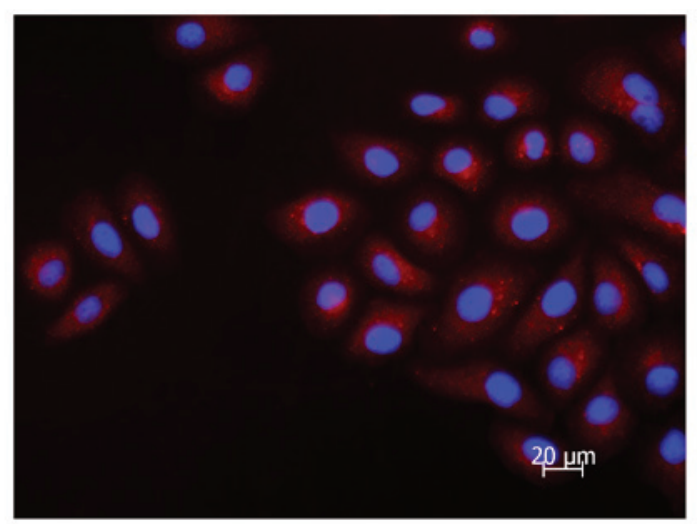

B

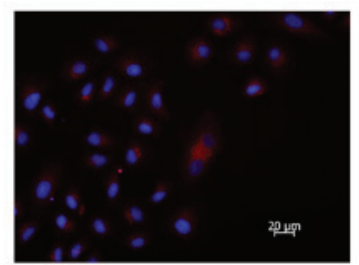

$\mathbf{F}$

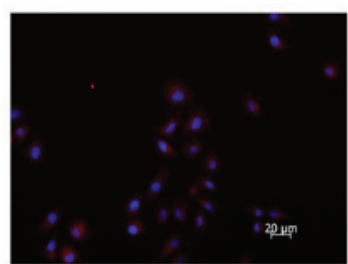

J

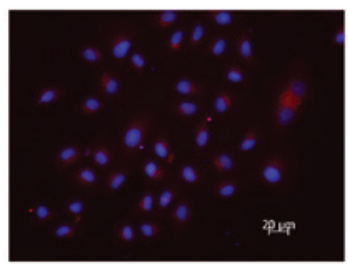

C

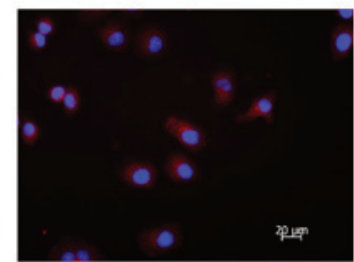

G

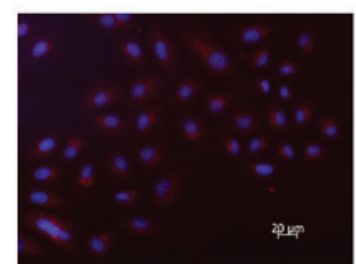

K

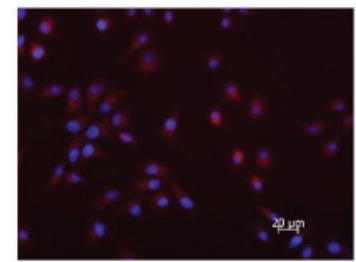

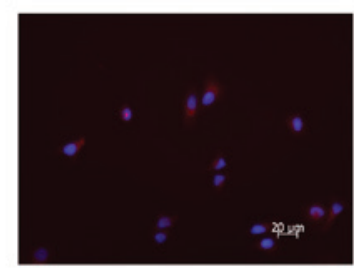

H

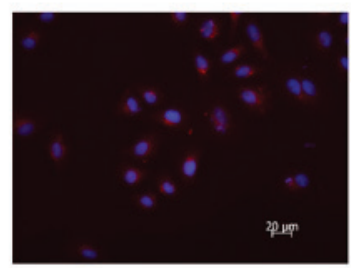

L

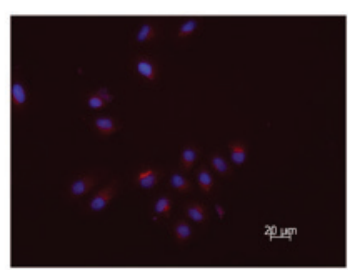

E

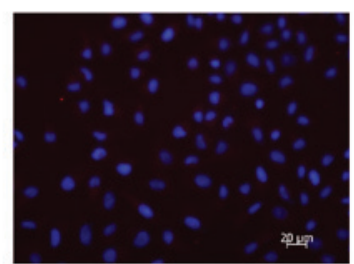

I

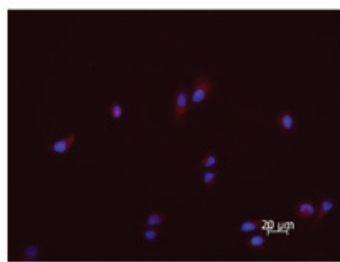

M

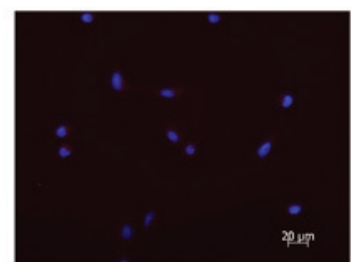

Figure 4. Microtubule-associated proteins 1A/1B light chain 3A expression and localization in SGC7901 human gastric cancer cells following treatment with PI3K(III)-RNAi-green fluorescent protein adenovirus and/or 5-fluorouracil. SGC7901 gastric cancer cells were incubated with PI3K(III)-RNAi-green fluorescent protein adenovirus (30 multiplicity of infection), NC-RNAi-green fluorescent protein adenovirus or 5-fluorouracil for 24 , 48 or 72 h, and then stained with $200 \mu \mathrm{mol} / 1 \mathrm{LC} 3$-cyanine 3. (A) $24 \mathrm{~h}$ after control treatment. (B) $24 \mathrm{~h}$ after NC-RNAi-green fluorescent protein adenovirus treatment. (C) $24 \mathrm{~h}$ after 5-fluorouracil treatment. (D) $24 \mathrm{~h}$ after PI3K(III)-RNAi adenovirus treatment. (E) $24 \mathrm{~h}$ after 5-fluorouracil and PI3K(III)-RNAi adenovirus treatment. (F) $48 \mathrm{~h}$ after NC-RNAi-green fluorescent protein adenovirus treatment. (G) $48 \mathrm{~h}$ after 5-fluorouracil treatment. (H) $48 \mathrm{~h}$ after PI3K(III)-RNAi adenovirus treatment. (I) $48 \mathrm{~h}$ after 5-fluorouracil and PI3K(III)-RNAi adenovirus treatment. (J) $72 \mathrm{~h}$ after NC-RNAi-green fluorescent protein adenovirus treatment. (K) $72 \mathrm{~h}$ after 5-fluorouracil treatment. (L) $72 \mathrm{~h}$ after PI3K(III)-RNAi adenovirus treatment. (M) $72 \mathrm{~h}$ after 5-fluorouracil and PI3K(III)-RNAi adenovirus treatment. Magnification, $\mathrm{x} 400 ; \mathrm{n}=3$. PI3K, phosphoinositide 3-kinase; RNAi, RNA interference.

of the chemotherapeutic agents kill cancer cells via the mitochondrial pathway. The mitochondrial pathway of apoptosis exerts a critical role. Mitochondrial permeability transition is an important event in the initiation of apoptosis (14). The findings from the present study revealed that the mitochondrial $\Delta \Psi$ collapsed following PI3K(III)-RNAi-GFP-AD treatment. Mitochondrial dysfunction is considered to be one of the most common and consistent phenotypes of cancer cells.

The majority of studies have indicated that autophagic inhibition sensitizes tumor cells to a wide spectrum of cancer therapies, while others have observed that treatment-induced tumor cell death requires the autophagic pathway (15).
Autophagic inhibition may snergize with other chemotherapeutic mechanisms to more effectively eliminate cancer cells. Recent studies supported this hypothesis, demonstrating promising effects in response to diverse chemotherapeutic agents, highlighting the possibility of autophagy-targeting adjuvant therapy in the treatment of cancer. The effects of autophagic inhibition treatment administered in combination with current anticancer therapies has been previously examined in various tumor models (16-18). In the present study, PI3K(III)-RNAi-GFP-AD exerted a greater effect when used in combination with 5-FU than when used alone. Thus, PI3K(III)-RNAi-GFP-AD inhibited the proliferation of SGC7901 gastric cancer cells, suppressed cell autophagy, 
A

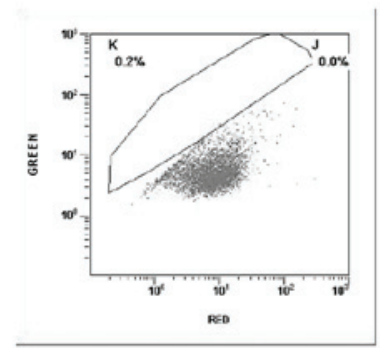

B

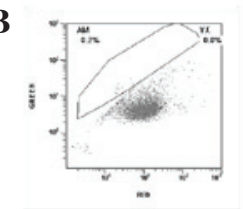

E

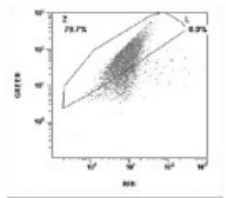

H

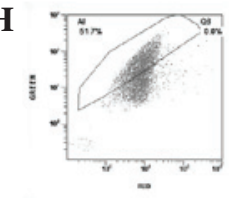

K

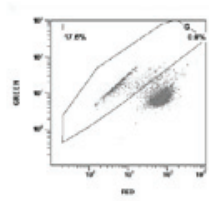

C

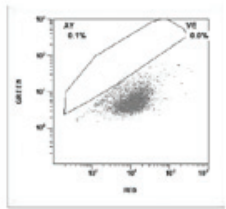

F

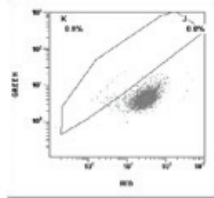

I

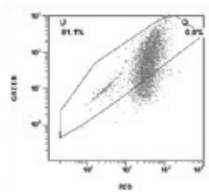

L

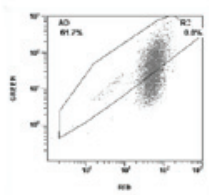

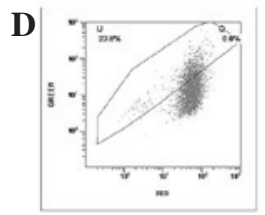

G

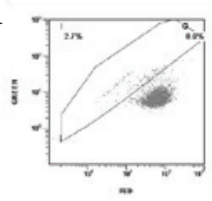

$\mathbf{J}$

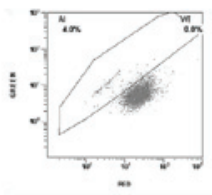

M

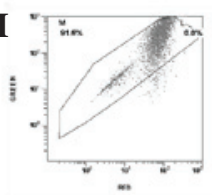

B

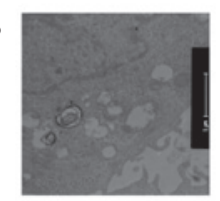

E

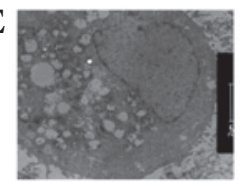

H

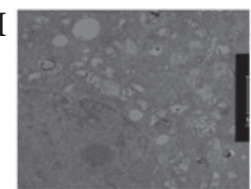

K

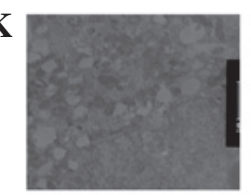

A
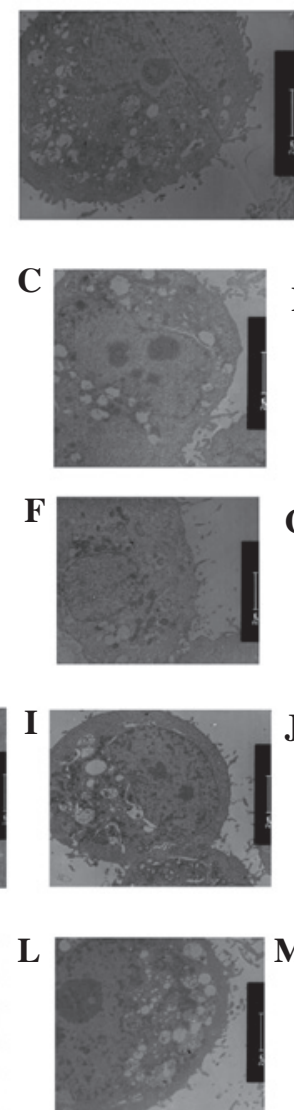
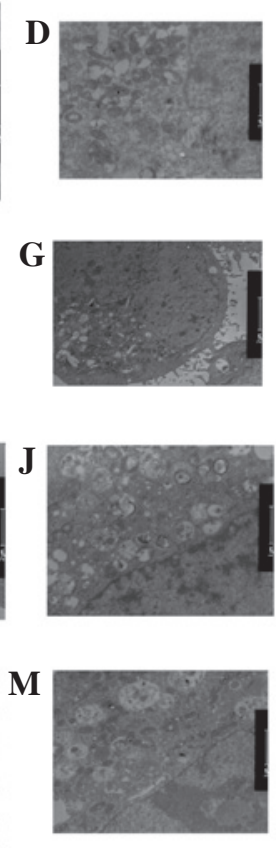

Figure 6. Ultrastructure of SGC7901 gastric cancer cells undergoing autophagy, apoptosis and necrosis following phosphoinositide 3-kinase (III)-RNA interference-green fluorescence protein adenovirus (30 multiplicity of infection) and/or 5-fluorouracil treatment. SGC7901 cells were incubated with phosphoinositide 3-kinase (III)-RNA interference-green fluorescence protein adenovirus (30 multiplicity of infection), NC-RNA interference-green fluorescence protein adenovirus or 5-fluorouracil for 24,48 or $72 \mathrm{~h}$. (A) $24 \mathrm{~h}$ control. (B) $24 \mathrm{~h}$ after NC-RNA interference-green fluorescence protein adenovirus treatment. (C) $24 \mathrm{~h}$ after 5 -fluorouracil treatment. (D) $24 \mathrm{~h}$ after phosphoinositide 3-kinase (III)-RNA interference adenovirus treatment. (E) $24 \mathrm{~h}$ after 5 -fluorouracil and phosphoinositide 3-kinase (III)-RNA interference adenovirus treatment. (F) $48 \mathrm{~h}$ after NC-RNA interference-green fluorescence protein adenovirus treatment. (G) $48 \mathrm{~h}$ after 5-fluorouracil treatment. (H) $48 \mathrm{~h}$ after phosphoinositide 3-kinase (III)-RNA interference adenovirus treatment. (I) $48 \mathrm{~h}$ after 5-fluorouracil and phosphoinositide 3-kinase (III)-RNA interference adenovirus treatment. (J) $72 \mathrm{~h}$ after NC-RNA interference-green fluorescence protein adenovirus treatment. (K) $72 \mathrm{~h}$ after 5-fluorouracil treatment. (L) $72 \mathrm{~h}$ after phosphoinositide 3-kinase (III)-RNA interference adenovirus treatment. (M) $72 \mathrm{~h}$ after 5 -fluorouracil and phosphoinositide 3-kinase (III)-RNA interference adenovirus treatment. $\mathrm{N}=3$. $\mathrm{NC}$, negative control.

5. Flow cytometric analysis of mitochondrial membrane poter indicated by green fluorescence ratio, following treatment of SGC7901 gastric cancer cells with PI3K(III)-RNAi-GFP-AD and/or 5-FU. The cells were incubated with PI3K(III)-RNAi-GFP-AD (30 multiplicity of infection), NC-RNAi-GFP-AD or 5-FU for the indicated time periods, and were then stained with JC-1 $(5 \mu \mathrm{mol} / 1)$. (A) $24 \mathrm{~h}$ after control treatment. (B) $24 \mathrm{~h}$ after NC-RNAi-GFP-AD treatment. (C) $24 \mathrm{~h}$ after 5-FU treatment. (D) $24 \mathrm{~h}$ after PI3K(III)-RNAi-AD treatment. (E) $24 \mathrm{~h}$ after 5-FU and PI3K(III)-RNAi-AD treatment. (F) $48 \mathrm{~h}$ after NC-RNAi-GFP-AD treatment. (G) $48 \mathrm{~h}$ after 5-FU treatment. (H) $48 \mathrm{~h}$ after PI3K(III)-RNAi-AD treatment. (I) $48 \mathrm{~h}$ after 5-FU and PI3K(III)-RNAi-AD treatment. (J) $72 \mathrm{~h}$ after NC-RNAi-GFP-AD treatment. (K) $72 \mathrm{~h}$ after 5-FU treatment. (L) $72 \mathrm{~h}$ after PI3K(III)-RNAi-AD treatment. (M) $72 \mathrm{~h}$ after 5-FU and PI3K(III)-RNAi-AD treatment. N=3. PI3K, phosphoinositide 3-kinase; RNAi, RNA interference; GFP, green fluorescent protein; 5-FU, 5-fluorouracil; $\mathrm{NC}$, negative control; $\mathrm{AD}$, adenovirus.

induced cell apoptosis and enhanced the chemosensitivity of 5-FU.

In conclusion, autophagic inhibition and apoptotic activation may markedly contribute to PI3K(III)-RNAi-GFP-AD-induced
SGC7901 cell death. In addition, autophagic inhibition may sensitize the cell chemosensitivity to 5-FU. Further investigation of upstream signal regulation of autophagy and apoptosis may provide novel insights with regard to the underlying mechanisms accommodating or contributing to autophagy and apoptosis, thereby unveiling novel strategies for tumor therapy.

\section{Acknowledgements}

This study was supported by the Natural Science Foundation of China (grant no. 81172348) and the Suzhou Science and Technology Development Foundation (grant nos. 2010SYS201031 and 2011SYSD2011092). 


\section{References}

1. Crew KD and Neugut AI: Epidemiology of gastric cancer. World J Gastroenterol 12: 354-362, 2006

2. Wu H, Yan Y and Backer JM: Regulation of class IA PI3Ks. Biochem Soc Trans 35: 242-244, 2007.

3. Yan Y and Backer JM: Regulation of class III (Vps34) PI3Ks. Biochem Soc Trans 35: 239-241, 2007.

4. Cantley LC: The phosphoinositide 3-kinase pathway. Science 296: 1655-1657, 2002.

5. Yu L, McPhee CK, Zheng L, Mardones GA, Rong Y, Peng J, Mi N, Zhao Y, Liu Z, Wan F, et al: Termination of autophagy and reformation of lysosomes regulated by mTOR. Nature 465: 942-946, 2010

6. Abedin MJ, Wang D, McDonnell MA, Lehmann U and Kelekar A: Autophagy delays apoptotic death in breast cancer cells following DNA damage. Cell Death Differ 14: 500-510, 2007.

7. Carew JS, Nawrocki ST, Kahue CN, Zhang H, Yang C, Chung L, Houghton JA, Huang P, Giles FJ and Cleveland JL: Targeting autophagy augments the anticancer activity of the histone deacetylase inhibitor SAHA to overcome Bcr-Abl-mediated drug resistance. Blood 110: 313-322, 2007.

8. Mizushima N, Levine B, Cuervo AM and Klionsky DJ: Autophagy fights disease through cellular self-digestion. Nature 451: 1069-1075, 2008.

9. Rubinsztein DC: The roles of intracellular protein-degradation pathways in neurodegeneration. Nature 443: 780-786, 2006.

10. Eisenberg-Lerner A, Bialik S, Simon HU and Kimchi A: Life and death partners: apoptosis, autophagy and the cross-talk between them. Cell Death Differ 16: 966-975, 2009.
11. Biederbick A, Kern HF and Elsässer HP: Monodansylcadaverine (MDC) is a specific in vivo marker for autophagic vacuoles. Eur J Cell Biol 66: 3-14, 1995

12. Hu Y, Xia XY, Pan LJ, et al: Evaluation of sperm mitochondrial membrane potential in varicocele patients using JC-1 fluorescent staining. Zhonghua Nan Ke Xue 15: 792-795, 2009 (In Chinese).

13. Kapp OH, Mainwaring MG, Vinogradov SN and Crewe AV: Scanning transmission electron microscopic examination of the hexagonal bilayer structures formed by the reassociation of three of the four subunits of the extracellular hemoglobin of Lumbricus terrestris. Proc Natl Acad Sci USA 84: 7532-7536, 1987.

14. Biswas G, Guha M and Avadhani NG: Mitochondria-to-nucleus stress signaling in mammalian cells: Nature of nuclear gene targets, transcription regulation, and induced resistance to apoptosis. Gene 354: 132-139, 2005.

15. Kondo Y, Kanzawa T, Sawaya R and Kondo S: The role of autophagy in cancer development and response to therapy. Nat Rev Cancer 5: 726-734, 2005.

16. Degtyarev M, De Mazière A, Orr C, et al: Akt inhibition promotes autophagy and sensitizes PTEN-null tumors to lysosomotropic agents. J Cell Biol 183: 101-116, 2008.

17. Lomonaco SL, Finniss S, Xiang C, et al: The induction of autophagy by gamma-radiation contributes to the radioresistance of glioma stem cells. Int J Cancer 125: 717-722, 2009.

18. Shingu T, Fujiwara K, Bögler O, et al: Stage-specific effect of inhibition of autophagy on chemotherapy-induced cytotoxicity. Autophagy 5: 537-539, 2009. 